\title{
Design, Synthesis and Biological Evaluation of some Chalcone Derivatives as Potential Pancreatic Lipase Inhibitors
}

\author{
Hoai-Anh Nguyen, Trong-Nhat Do, Van-Dat Truong, Khac-Minh Thai, Ngoc-Chau Tran, Thanh-Dao Tran ${ }^{\bowtie}$ \\ Faculty of Pharmacy, Ho Chi Minh City University of Medicine and Pharmacy \\ 41 Dinh Tien Hoang Street, District 1, Ho Chi Minh City, Vietnam
}

\begin{abstract}
Obesity is a growing global health problem, but few drugs are available for the treatment of obesity. Several classes of compounds have been studied and demonstrated for the human lipase inhibition activity - a target in obesity prevention. This study was about design, synthesis and biological evaluation of some synthetic chalcones as pancreatic lipase inhibitors.

FlexX software integrated in LeadIT was used for molecular docking studies of 66 chalcone derivatives. 6 derivatives with low docking scores (good binding affinity) were selected for synthesis using both classical and microwave-assisted Claisen-Schmidt condensation reactions.

Biological evaluation on pancreatic lipase indicated that some chalcones showed good lipase inhibition activities and that there were correlations between in silico model and biological activities. These presented the possibility to apply virtual screening tools in finding potential agents with high obesity-prevention capacity.
\end{abstract}

Keywords: lipase inhibitory activity, chalcone,

\section{INTRODUCTION}

Obesity has been cited as a major social issue, both in developed and developing countries. According to statistics by WHO, the number of overweight adults reached 1.4 billion in 2008 . Of these 500 million people was identified as clinical obese and the rate has nearly doubled since 1980. Obesity would be no cause for patients' concern if it was not the reason behind the surging risks of dyslipidemia, diabetes mellitus type 2, coronary artery disease, stroke, as well as musculoskeletal and airway disorders, prostate cancer, etc [5]. There is a huge potential market for anti-obesity drugs [4], which contributed to roughly 2-6\% healthcare expenditure in many developed nations and it was estimated to reach $\$ 3,7$ billion in 2008 . There is, however, quite a few active substances approved by FDA for long-term obesity treatment like orsilat (pancreatic lipase inhibitor), lorcaserin (serotonin $2 \mathrm{C}$ receptor activator) alongside with short-term drugs like phentermine, zonisamide [5], [9].

Drugs are used for patients with BMI over 30 or over 27 in case they suffer from some accompanying diseases such as hypertension, insulin resistance [20]. Many indications was used to be adopted for many years. Nevertheless, almost the anti-obesity drugs allowed on the market has been withdrawn for their adverse effects. Numerous drugs today (with new indications or combination) are under study, of which lorcaserin (Belviq ${ }^{\circledR}$ ) is the first substance within 13 years to be approved by FDA on June 27 this year [18]; and phentermine/topiramate combination $\left(\right.$ Qsymia $\left.^{\circledR}\right)$ was adopted by FDA for the long-term obesity treatment on July, 17, 2012 [19].

Orlistat (prescription branded-name Xenical ${ }^{\circledR}$, Roche and OTC low-dose Alli ${ }^{\circledR}$, GSK) is the long-term anti-obesity drug approved by FDA in the US (1999). Orsilat is not misused because of its inability to absorption $(<1 \%)$ and it is also safe for central nervous system. As a 
pancreatic lipase inhibitor, orsilat is responsible for lowering fat absorption by more than $30 \%$ and is not associated with trypsin, chymotryptrin and phospholipase. Orsilat also inhibits gastric lipase, carboxyesterase and phospholipase A2 [1].

Pancreatic lipase is a common target for anti-obesity drug research. Pancreatic lipase (triacylglycerol acyl hydrolase) plays an essential role in the digestion of triacylglycerols. Excreted in the duodenum, pancreatic lipase hydrolyzes $50-70 \%$ fat (at a maximum rate of 140 grams per minute) in the presence of colipase and $\mathrm{Ca}^{2+}$. Several classes of substances exhibit pancreatic lipase inhibitory activity, including flavonoids, saponins [4],[17], and substances of microbial sources such as lipstatin, panclicins, etc [2].

The chalcone derivatives (of flavonoid group) are both naturally occurred and synthesized in a straightforward fashion in laboratory. As human safety is revealed [3], chalcone analogs are given priority for the investigation into pancreatic lipase inhibitory activity. This opens up new directions in bioactivity studies at Department of Pharmaceutical Chemistry, University of Medicine and Pharmacy in HCMC, besides prostaglandin E2 inhibitory [15], antioxidant and antifungal activity [6], antimicrobial activity of chalcones analogs and heterocyclic chalcones [14].

\section{MATERIALS AND METHODS}

\section{Docking}

In terms of properties of protein structure collected from database banks and the aim of evaluating the possibility of human lipase inhibitor, protein of PDB code 1LPB fixes requirements for a human lipase model, having targeted ligand is the inhibitor in the active site of enzyme, with high resolution of $2.46 \AA$.

Protein 1LPB was protonated by MOE 2008.10. Then, 1LPB's pocket was created by LeadIT 2.1.1. Water molecules and ions were removed. Binding site chosen was of $6.5 \AA$ surrounding co-crystallized ligand-methoxyundecylphosphinic acid (MUP). Binding site includes paramount amino acids in triple catalysis Ser152, Asp176. Chalcone formulas were docked to the target protein by LeadIT 2.1.1. Co-crystallized ligand also went through redock (the technique of docking co-crystallized ligand in the active site of enzyme) with similar parameters; based on the values of root-mean-square deviation (RMSD) it is likely to evaluate the precision of ligand preparation procedure and docking method.

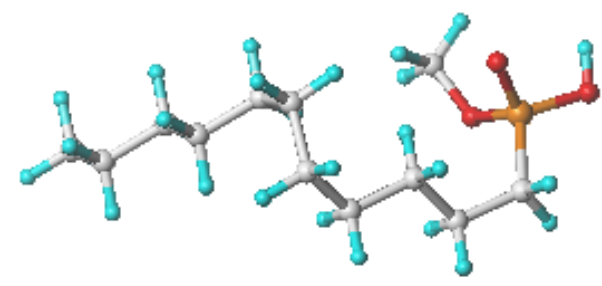

Figure 1. 3D structure of methoxyundecylphosphinic acid (MUP)

Synthesis - microwave-assisted method

To a $100 \mathrm{~mL}$ Erlenmeyer flask containing an acetophenone derivative $(5 \mathrm{mmol})$ and a benzaldehyde derivatie $(5.5 \mathrm{mmol})$ dissolved in $96 \%$ ethanol $(6 \mathrm{~mL}), 60 \% \mathrm{KOH}$ solution $(2$ $\mathrm{mL}$ ) was added dropwise. The reaction mixture was immersed in an ice bath, stirred frequently and placed in $300 \mathrm{~W}$ microwave oven within 6-10 minutes. Time for every reaction was set in 30 seconds, the flask was then allowed to cool before the next turn, in case ethanol was overheated, evaporated vigorously and the mixture was extruded from the flask or caught fire. The process was monitored by thin layer chromatography. After completion, the mixture 
was allowed to stand to room temperature and laid overnight. The mixture was acidified by $10 \% \mathrm{HCl}$ solution, cooled and stirred frequently to $\mathrm{pH} 4$. The obtained precipitation was filtered, washed to neutralized $\mathrm{pH}$. The product was recrystallized by ethanol or ethanol-water mixture [9].

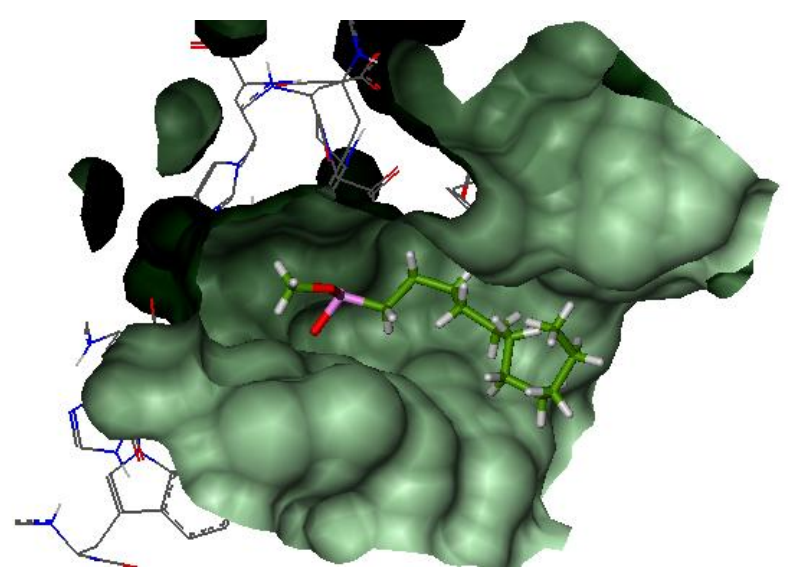

Figure 2. Co-crystallized ligand in the active site of enzyme

\section{Porcine Pancreatic Lipase Inhibiton Assay}

Porcine pancreatic lipase is often the prevalent choice for pancreatic lipase inhibitory activity, since there is roughly a 85 percentage of homologous genes between human and porcine [11]. The assay also fits the cost and the goal of this study. Based on the absorbance variation of the reaction mixture, emulsified triolein was used as a substrate for the lipase inhibition assay [7]. The substrate, the inhibitor and lipase were mixed and absorbance at a wavelength of 450 $\mathrm{nm}$ was monitored, the time $\mathrm{T}_{0}$ was recorded. The reaction was kept in a suitable condition $\left(37^{\circ} \mathrm{C}\right)$ and absorption was recorded at $\mathrm{T}_{30}$.

Variation of the absorbance is designated as $\left[\mathrm{A}_{450}\left(\mathrm{~T}_{0}\right)-\mathrm{A}_{450}\left(\mathrm{~T}_{30}\right)\right]$, for both the test and the blank sample. \% Inhibition was calculated as following (13):

$$
\% \text { Inhibition }=\frac{\Delta A_{450 \text { blank }}-\Delta A_{450 \text { test }}}{\Delta A_{450 \text { blank }}} \times 100 \%
$$

\section{RESULTS AND DISCUSSIONS}

\section{Docking}

The result of docking with 66 chalcones and four other agents with $\mathrm{IC}_{50}$ values on protein 1LPB by LeadIT 2.1.1 after formulas were optimized and prepared by Sybyl-X 1.1. 10 (Formulas with the highest docking scores were illustrated in table 1)

Table 1. Doking results of 10 formulas with the highest docking score

\begin{tabular}{ccllc}
\hline No & Structures & Scores & \multicolumn{1}{c}{ Hydrogen Bonds } & $\boldsymbol{\pi}-\boldsymbol{\pi}$ Bonds \\
\hline 1 & S16 & $-26,0210$ & Gly76, Asp79, Ser152, His263 & Phe215 \\
\hline 2 & S51 & $-25,1390$ & Ser152, His263, Gly76, Asp79 & \\
\hline 3 & S27 & $-24,9490$ & Ser152, His151, Gly76 & \\
\hline 4 & S28 & $-24,8970$ & Tyr114 & Tyr114 \\
\hline 5 & S35 & $-24,8510$ & Ser152, Gly76 & Tyr114 \\
\hline 6 & S15 & $-24,8140$ & Ser152, Phe215, Gly76, Asp79 & Tyr114 \\
\hline 7 & S3 & $-24,436$ & Ser152, His263, Gly76, Asp79 & Tyr114 \\
\hline 8 & S56 & $-23,9090$ & His151 & Phe215 \\
\hline 9 & S43 & $-23,7900$ & Ser152, Phe77 & \\
\hline
\end{tabular}

Interactions between some ligands and receptors were illustrated in Figure 1 and Figure 2. 


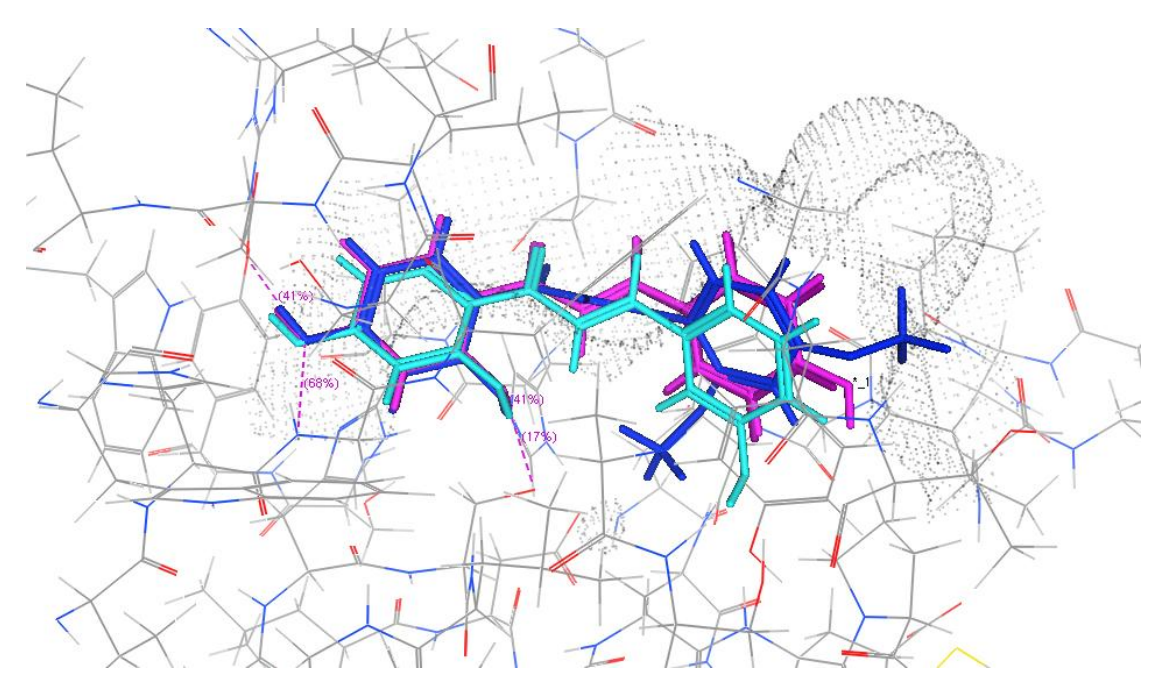

Figure 3. 3D interactions between four ligands (S51, S19, S15, S3) and their receptor.

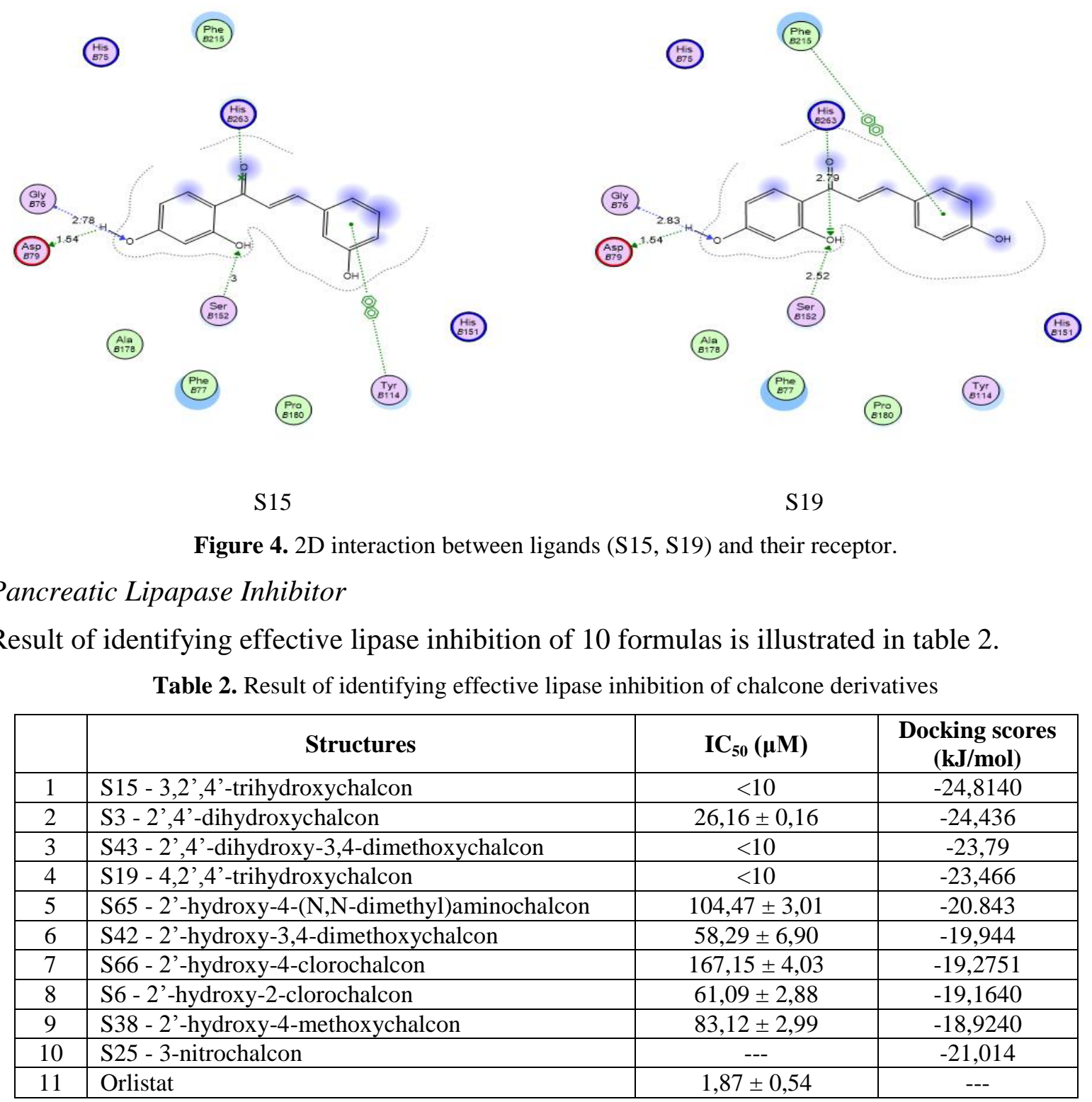

Correlations between docking scores and $\mathrm{IC}_{50}$ of biologically evaluated derivatives are presented in Figure 5, in which values of $\mathrm{IC}_{50}$ are presented in forms of $\mathrm{pIC}_{50}$. 


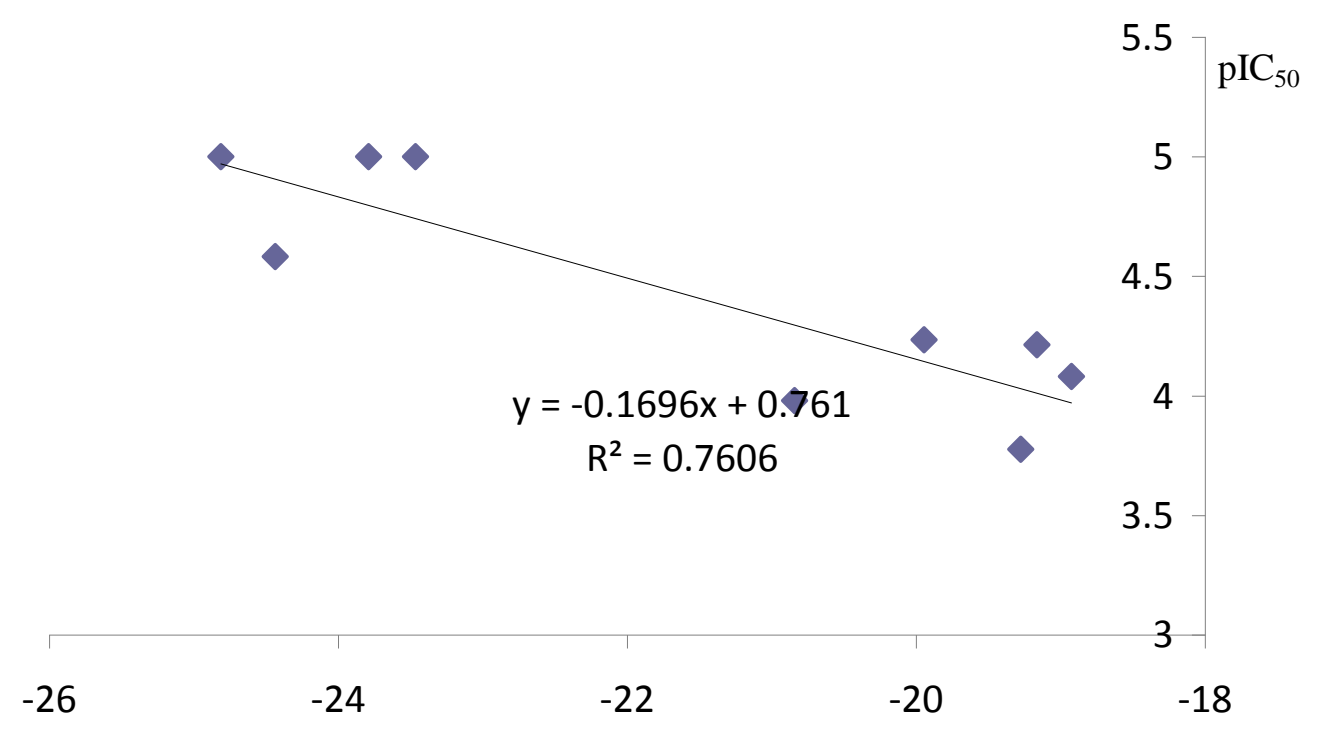

Figure 5. Correlation between $\mathrm{IC}_{50}$ and docking scores

Based on ligand-receptor interactions and substances with $\mathrm{IC}_{50}<10 \mu \mathrm{M}$, some structure activity relationship of chalcones were proposed. Carbonyl group in chalcone structure is important for its bonding to amino acids of threesome catalysis. Having hydroxyl substituents at 2', 4' positions showed better potential lipase inhibitor than having only one hydroxyl substituent at 2' position. Amino group at 4' position shows good effect on inhibiting enzyme when having additional hydroxyl group or nitro group at position 3 on ring B. In addition, positions of substituents on ring B such as hydroxyl at position 3 (S15) have better potential comparing with hydroxyl group at position 4 (S19).

For the purpose of selecting some of chalcone derivatives by using docking model running on computer, this research is hoped to find out potential chalcone compounds which are able to resist pancreatic lipase activities. Based on docking outcomes, it is found that chalcones with many hydroxy or amino substituents not only lead to good cohesion results but also be capable of creating bonds with some critical amino acids. Among the derivatives used in the docking model, some of them were studied for many other effects such as anti-inflammatory, anti-bacterial, anti- tumor abilities (S19, S14, S18...) [12],[15]. A new point of this research is that in the list of used compounds, there are some derivatives containing two hydroxy substituents at meta-position (resorcinol derivatives). These derivatives bring about favorable docking results. However, in terms of synthesis, the two - $\mathrm{OH}$ substituents at meta-position are oxidized easily in alkaline environment at appropriate temperature. Comparing the docking results to $\mathrm{IC}_{50}$ of published compounds with $\mathrm{R}^{2}=0.986$ proves that there is correlation between docking results and testing results. This is to say, the compounds with lower $\mathrm{IC}_{50}$ lead to lower negative values in docking result.

When it comes to the synthesis process, in the trend of "green chemistry", the research is chose to be ran in a safe method reducing poisonous solvents (in order to protect the environment), using microwave in a short period of time (for purpose of saving electricity and water). Nevertheless, using microwave-assisted Claisen-Schmidt synthesis reaction requires intense caution in working with equipment than heating under reflux on magnetic stirrers. The solvent for microwave reaction is ethanol-water, hence when microwave energy is transmitted to the reaction's mixture; ethanol is boiled easily and creates a high vapor pressure, resulting in overflow the reaction's mixture. Therefore, the reaction needs to be carried out in short periods of time (from 15 to 30 seconds) and alternately be cooled with or without inert gas environment $\left(\mathrm{N}_{2}\right.$, argon as the condition of our laboratory). Among a number of compounds 
which is chosen to synthesize, isoliquiritigenin (S19; 2',4,4'-trihydroxychalcone) - a natural compound extracted from liquorice - was studied for many different effects and anti-lipase ability $\left(\mathrm{IC}_{50}=7,3 \mu \mathrm{M}\right.$ [3]). Because it is a chalcone derivative containing three hydroxy substituents, to synthesize S19 all of factors such as temperature, alkaline environment and time of reaction need to be controlled in the case of using Claisen-Schmidt reaction with microwave or hydroxyl substituent in the chain of acetophenone must be protected against oxidation reactions and coupling reactions of 2'-hydroxy.

Results of testing anti-lipase activity show that many compounds are potential on this target. Based on Figure 5, with $\mathrm{R}^{2}=0.7606$ there is a correlation between docking model outcomes and results of active tests. The active test carrying out with ten compounds brings about a result that 9 of them have anti-lipase ability. Specially, S25's ability cannot be determined because of its insolubility at necessary concentration. Meanwhile, other multiple hydroxy substituent compounds such as S15, S43, and S19 have $\mathrm{IC}_{50}$ value under $10 \mu \mathrm{M}$. $\mathrm{IC}_{50}$ value of published compounds, $\mathrm{S} 19$ ( $\mathrm{IC}_{50} 7,3 \mu \mathrm{M}$ [3], experimental value $<10 \mu \mathrm{M}$ ) and orlistat $\left(\mathrm{IC}_{50}\right.$ $0,5 \mu \mathrm{M}$ [10], experimental value $=1,87 \mu \mathrm{M}$ ) demonstrate that chalcone synthetic derivatives are possible to inhibit lipase activity. Thanks to the research, chalcone derivatives are proven to have the anti-pancreatic lipase, applying into obesity treatment.

\section{CONCLUSIONS}

In this study, we screened 66 chalcone derivatives, synthesized 6 biologically potential derivatives and evaluated 9 derivatives for their lipase inhibitory activities. The methods were mainly composed of three stages. The results indicated that there were correlations between in silico models and biological activities, hence also presented the possibility to apply virtual screening tools in finding potential agents with high obesity-prevention capacity.

\section{Acknowledgments}

This work was supported by the Vietnam's National Foundation for Science and Technology Development - NAFOSTED (Grant \# 104.01.2012.78 to Thanh-Dao Tran).

\section{References}

1. Al-Suwailem K., Al-Tamimi A. S. (2006), "Safety and mechanism of action of orlistat (tetrahydrolipstatin) as the first local antiobesity drug," Journal of Applied, 2(4), 205-208.

2. Birari R. B., Bhutani K. K. (2007), "Pancreatic lipase inhibitors from natural sources: unexplored potential," Drug discovery today, 12(19-20), 879-89.

3. Birari R. B., Gupta S., Mohan C. G., Bhutani K. K. (2011), "Antiobesity and lipid lowering effects of Glycyrrhiza chalcones: experimental and computational studies," Phytomedicine, 18(8-9), 795-801.

4. De la Garza A. L., Milagro F. I., Boque N., Campión J., Martínez J. A. (2011), "Natural inhibitors of pancreatic lipase as new players in obesity treatment," Planta Medica, 77(8), 773-85.

5. Derosa G. (2012), "Anti-obesity drugs: a review about their effects and their safety," Expert Opinion on Drug Safety, 11(3), 459-471.

6. Doan T., Tran T-D. (2011), "Synthesis, Antioxidant and Antimicrobial Activities of a Novel Series of Chalcones, Pyrazolic Chalcones, and Allylic Chalcones," Pharmacology \& Pharmacy, 2, 282-288.

7. Etoundi C. B., Kuaté D., Ngondi J., Ben J. (2010), "Anti-amylase, anti-lipase and antioxidant effects of aqueous extracts," Journal of Natural Products, 3, 165-171.

8. Jincheng Y., Yongbo Y., Lijun L. (2011), "Synthesis of Isoliquiritigenin and Its 
derivatives," Journal of Ningxia University, 32(3), 255-257.

9. Kang J. G., Park C.-Y. (2012), "Anti-Obesity Drugs: A Review about Their Effects and Safety." Diabetes \& metabolism journal, 36(1), 13-25.

10. Lee E., Lee S., Chung B., Cho J., Lee I. (2010), "Pancreatic Lipase Inhibition by CGlycosidic Flavones Isolated from Eremochloa ophiuroides," Molecules, 15, 8251-8259.

11. Lowe M., Rosenblum J. (1989), "Cloning and characterization of human pancreatic lipase cDNA," Journal of Biological Chemistry, 264, 20042-20048.

12. Minh-Tri Le, Thi-Ngoc-Phuong Huynh (2011). Pharmaceutical Chemistry 1, Medical Publisher, Hanoi, p. 58-88.

13. Schramm O. (2006), Multicomponent Heterocycle Syntheses Based upon Sonogashira Coupling-Isomerization, $\mathrm{PhD}$, Ruprecht-Karls University, Heidelberg.

14. Tran T., Nguyen T., Do T., Huynh T. (2012), "Synthesis and Antibacterial Activity of Some Heterocyclic Chalcone Analogues Alone and in Combination with Antibiotics," Molecules, 17, 6684-6696.

15. Tran T., Park H., Kim H., Ecker G. (2009), "Inhibitory activity of prostaglandin E2 production by the synthetic 2'-hydroxychalcone analogues: Synthesis and SAR study," Bioorganic \& Medicinal Chemistry Letters, 19, 1650-1653.

16. Yong-Bo Y., Shu-Juan L., Li-Jun L. (2010), "Characterization of Interaction between Synthetic Isoliquiritigenin and Bovine Serum Albumin by Fluorescence Quenching," Chinese Journal of Natural Medicines, 8(5), 388-342.

17. Yun J. W. (2010), "Possible anti-obesity therapeutics from nature--a review," Phytochemistry, 71(14-15), 1625-1641.

18. FDA, (2012), FDA approves Belviq to treat some overweight or obese adults. FDA. 23/07/2012,http://www.fda.gov/NewsEvents/Newsroom/PressAnnouncements/ucm30999 3.htm

19. FDA, (2012), "FDA approves weight-management drug Qsymia," FDA. 23/07/2012, http://www.fda.gov/NewsEvents/Newsroom/PressAnnouncements/ucm312468.htm

20. Merck \& Co., (2008), "Obesity and the Metabolic Syndrome," Merck Manual for Healthcare Frofessionals. 15/07/2012, http://www.merckmanuals.com/professional/print/nutritional_disorders/obesity_and_the_ metabolic_syndrome/obesity.html 